\title{
Seroprevalence of Cysticercosis in Children and Young Adults Living in a Helminth Endemic Community in Leyte, the Philippines
}

\author{
Jin-Mei Xu, ${ }^{1}$ Luz P. Acosta, ${ }^{2}$ Min Hou, ${ }^{1}$ Daria L. Manalo, ${ }^{2}$ Mario Jiz, ${ }^{2}$ Blanca Jarilla, ${ }^{2}$ \\ Archie O. Pablo, ${ }^{2}$ Remigio M. Ovleda, ${ }^{2}$ Gretchen Langdon, ${ }^{3}$ Stephen T. McGarvey, ${ }^{4}$ \\ Jonathan D. Kurtis, ${ }^{3,5}$ Jennifer F. Friedman, ${ }^{3,6}$ and Hai-Wei Wu ${ }^{1,3,6}$ \\ ${ }^{1}$ Department of Pathogen Biology, Nanjing Medical University, Nanjing, Jiangsu 210029, China \\ ${ }^{2}$ Department of Immunology, Research Institute for Tropical Medicine, Department of Health, Manila 1781, Philippines \\ ${ }^{3}$ Center for International Health Research, Rhode Island Hospital, Providence, RI 02906, USA \\ ${ }^{4}$ International Health Institute, Brown University School of Medicine, Providence, RI 02912, USA \\ ${ }^{5}$ Department of Pathology and Laboratory Medicine, Brown University Medical School, Providence, RI 02912, USA \\ ${ }^{6}$ Department of Pediatrics, Brown University Medical School, Providence, RI 02912, USA
}

Correspondence should be addressed to Hai-Wei Wu, haiwei_wu@brown.edu

Received 17 August 2009; Revised 20 October 2009; Accepted 19 January 2010

Academic Editor: Luis Eduardo Cuevas

Copyright ( 92010 Jin-Mei Xu et al. This is an open access article distributed under the Creative Commons Attribution License, which permits unrestricted use, distribution, and reproduction in any medium, provided the original work is properly cited.

Cysticercosis is a significant public health problem in countries where pigs are raised for consumption and remains an important cause of neurological disease worldwide. The Philippines is considered an endemic area for cysticercosis because cases in both humans and pigs have been reported; however, epidemiologic information stays limited. We conducted a pilot survey of the seroprevalence of human cysticercosis in a village in Leyte, the Philippines, by measuring antibody specific for Taenia solium cystfluid antigen. There were 497 subjects aged 7-30 years in our study and most subjects were infected with one or more helminths. The overall cysticercosis seroprevalence in this population was $24.6 \%$ (95\% CI: $20.82 \% \sim 28.58 \%$ ) with no significant difference based on age, sex, or other helminth coinfection status. Although the sample may not be representative of the whole community, the findings suggest that cysticercosis is a significant, but underrecognized public health concern in the Philippines.

\section{Introduction}

Human cysticercosis is caused by infection with the larvae (cysticerci) of Taenia solium, a cestode (tapeworm) transmitted among humans and between humans and pigs. Humans acquire tapeworm infection from eating raw or undercooked pork meat containing T. solium cysticerci. When ingested, cysticerci are activated by stomach acid, pass into the duodenum, and develop into adult tapeworms. The tapeworm body consists of many proglottids, each containing approximately 50,000-60,000 eggs [1]. Both humans and pigs can develop cysticercosis if they ingest $T$. solium eggs passed in human stool directly, or from consumption of food or water contaminated with eggs (fecal-oral transmission). Human cysticercosis can also occur by autoinoculation or reverse peristalsis of eggs in individuals with $T$. solium tapeworms [2]. The clinical presentation of cysticercosis is nonspecific and varies depending on the location, number, and stage of cysts. The most frequently reported locations are skin, skeletal muscle, heart, eye, and most importantly, the central nervous system, causing neurocysticercosis (NCC) [3-7].

Cysticercosis is endemic in Africa, Asia, and Latin America [8]. It is mainly transmitted in areas where pigs range freely, sanitation is poor, human feces are used as fertilizer, education is low, and meat inspection is absent or inadequate, and thus is strongly associated with poverty and smallholder farming. In recent years, a growing number of cysticercosis cases have been reported in more developed countries as a result of increasing migration and tourism 
[2, 9-13]. Theoretically, cysticercosis is straightforward to prevent and control; however, it has not been eliminated and remains neglected in both the endemic developing countries $[8,14]$ and developed countries [15].

At present, epidemiological surveys of cysticercosis have not been conducted in many endemic areas due to the lack of availability and cost of the diagnostic methods [16]. Diagnostic approaches for cysticercosis include subcutaneous nodule biopsy, neuroimaging, and serological tests [8]. Neuroimaging, that is, computerized tomography (CT) and magnetic resonance imaging (MRI), are very useful for diagnosis of cysticercosis, but they are inaccessible in many poor endemic areas of the world [17]. Immunodiagnostic methods for cysticercosis, which detect parasite antigens or host antibodies to parasite antigens, have been developed and improved greatly in recent years and allow identification of endemic communities where prevention and control measures should be implemented $[18,19]$.

The Philippines is a developing country whose economy relies mainly on agriculture and is considered an endemic area because both human [20] and porcine cysticercosis [21] have been reported. However, information on the epidemiology of this disease is still quite limited in the Philippines. To appropriately target scarce health care resources, detailed, community based studies of the prevalence of cysticercosis in pig farming areas of the Philippines are necessary. Therefore, we conducted a human seroprevalence study of cysticercosis using a commercially available kit which detects antibodies to cysticercal antigens in a village in Leyte where schistosomiasis and geo-helminths are coendemic. Our results indicate that human cysticercosis is an underappreciated infection in this area.

\section{Materials and Methods}

2.1. Ethics Statement. The study was approved by Institutional Review Board at Brown University and at the Philippines Research Institute of Tropical Medicine. Written, informed consent was obtained from each adult participant or from the parents of minors.

2.1.1. Study Design. This study was conducted in Macanip, a $S$. japonicum-endemic rice-farming village in Leyte, the Philippines. The study area is endemic for both S. japonicum and geo-helminths. The current cross-sectional serologic survey of cysticercosis was performed in subjects who were eligible for a longitudinal treatment-reinfection study of schistosomiasis $[22,23]$. S. japonicum infected individuals were enrolled in the study during October 2002 if they lived primarily in the study village, and were not pregnant or lactating, and provided informed consent. The study sample consisted of $422 \mathrm{~S}$. japonicum infected individuals aged 7 to 30 years and individuals aged 7 to 18 years $(n=75)$ who were not infected with $S$. japonicum were recruited as control subjects. The prevalence of infection with $S$. japonicum in this age range was $60.0 \%$ in the community. Due to the overall study design, the sample for serologic survey of cysticercosis has a much higher proportion of S. japonicum infection than the age-specific or general prevalence in the community [23]. For each subject, infections of S. japonicum, A. lumbricoides, T. tricuria, and hookworms were determined through Kato-Katz examination of 2 slides prepared from each of 3 stool samples. All participants received treatment with a split dose of $60 \mathrm{mg}$ praziquantel/Kg of body weight after baseline blood collection and physical examination.

2.1.2. Blood Collection. Prior to treatment for schistosome infection, blood was collected into Vacutainer tubes (Becton Dickinson and Company, Franklin Lakes, NJ) for all subjects. Serum was prepared, aliquoted, and stored at $-80^{\circ} \mathrm{C}$ until assayed.

2.1.3. ELISA Assay. All serum samples were tested by indirect ELISA assay to quantify the level of antibody reacting with T. solium cyst-fluid antigen using a commercial diagnostic kit (Shenzhen Combined Biotech Co. Ltd., China). The reported sensitivity and specificity of this assay kit are $92.5 \%$, and $100 \%$, respectively, with no false positives occurring in samples from patients with trichinosis, fascioliasis, paragonimiasis, and nonparasitic encephalopathy.

The ELISA assay was performed following the instructions and using the diluents supplied with the kit. Briefly, serum samples were diluted $1: 8$ in kit diluent and transferred into wells $(100 \mu \mathrm{L} /$ well $)$ of the plates that are precoated with $T$. solium cyst-fluid antigen followed with blocking by bovine serum. Then, the plates were incubated at $37^{\circ} \mathrm{C}$ for half an hour and washed five times with washing buffer. Horseradish peroxidase (HRP)-labeled goat antihuman $\operatorname{IgG}(\mathrm{H}+\mathrm{L})$ antibody $(100 \mu \mathrm{L} /$ well $)$ was added and incubated at $37^{\circ} \mathrm{C}$ for half an hour followed by washing five times. After adding chromogenic substrate TMB $\left(3,3^{\prime}, 5,5^{\prime}-\right.$ tetramethylbenzidine) $(50 \mu \mathrm{L} /$ well $)$, plates were incubated at $37^{\circ} \mathrm{C}$ for half an hour and the reactions were stopped with $2 \mathrm{M} \mathrm{H}_{2} \mathrm{SO}_{4}$. OD values were recorded at $450 \mathrm{~nm}$ with reference wavelength of $620 \mathrm{~nm}$ by the ELISA microplate reader (Clinibio 128C, ASYSHitch GmbH, Austria).

The serum sample of each study subject was assayed in triplicate. All control sera were run on each plate in duplicate. One negative control serum provided by the kit was diluted 1:8 while a standard reference serum prepared using pooled sera from ten cysticercosis patients was diluted serially 2 -fold from $1: 8$ to $1: 1024$. Based on the ODs of standard reference sera on each ELISA plate, a standardization method I-STOD (improved-optical density standardization) was used to transform the OD value of all serum samples into standardized antibody concentration as arbitrary units for analysis of antibody level in the population [24]. All sera with OD values greater than 2.1 times of mean $\mathrm{OD}$ value of the negative control serum were defined as antibody-positive according to the kit instruction.

2.1.4. Statistical Analysis. All statistical analysis was performed using STATA 7.0 software. Because the distribution of specific antibody concentration in the study population was right skewed, natural logarithm transformation 
TABLE 1: The prevalence of the four helminth infections in the study sample.

\begin{tabular}{lcccc}
\hline Category & Variable & $\begin{array}{c}\text { Number of } \\
\text { positives }\end{array}$ & $\begin{array}{c}\text { Percent } \\
\text { Positive }(\%)\end{array}$ & $\begin{array}{c}95 \% \mathrm{CI}^{*} \\
(\%)\end{array}$ \\
\hline \multirow{3}{*}{ Helminth } & S. japonicum & 422 & 84.9 & $81.5-88.0$ \\
infected & Ascaris & 353 & 71.0 & $66.8-75.0$ \\
& Trichuris & 460 & 92.6 & $89.9-94.7$ \\
& Hookworm & 259 & 52.1 & $47.6-56.6$ \\
\hline \multirow{3}{*}{ Multiple } & None & 2 & 0.4 & $0.5-1.5$ \\
helminth & One & 31 & 6.2 & $4.3-8.7$ \\
infections & Two & 100 & 20.1 & $16.7-23.9$ \\
& Three & 193 & 38.8 & $34.5-43.3$ \\
& Four & 171 & 34.4 & $30.2-38.8$ \\
\hline
\end{tabular}

* confidence interval.

$(\ln ($ concentration +1$))$ was performed for each concentration value before multivariate regression analysis. Multivariate linear regression analysis was used to detect the influence of sex, age, and coinfection status for four different helminths (S. japonicum, A. lumbricoides, T. tricuria, and hookworms categorized as Yes/No infection), on the antibody level (natural logarithm transformationed, $(\ln ($ concentration +1$))$, specific to T. solium cyst-fluid antigen. Multivariate logistic regression analysis was performed to evaluate the relationship between sex, age, or different helminth infections (categorized as Yes/No infection) and T. solium seropositivity. Comparison of proportions between different groups was performed using chi-squared test.

\section{Results}

3.1. Description of Study Population. The study sample consisted of 497 individuals, of which, 310 were males and 187 were females. The mean age of this sample was 14.9 years with a standard deviation of 6.0 years. Most subjects were infected with one or more helminths. The prevalences of the four helminths infections determined by stool examination (S. japonicum, A. lumbricoides, T. tricuria, and hookworms) were all above $50 \%$, and $93.4 \%$ of the subjects were infected with multiple helminths (Table 1). No T. solium carrier was detected using the coprological examination method.

\subsection{Seroprevalence of Cysticercosis in the Study Sample}

3.2.1. Distribution of Taenia solium Cyst-Fluid AntigenSpecific Antibody. The distribution of T. solium cyst-fluid antigen-specific $\operatorname{IgG}$ (concentration relative to the standard reference serum) in the study population was right skewed ranging from 0 to 3.015 unit (Figure 1). In accordance with the cutoff defined by 2.1 times of mean I-ODST unit of the negative control serum, the cut-off value for positive antibody concentration was 0.045 . In multivariate linear regression analyses, specific antibody level was not related with age or sex. No relationship between $T$. solium cystfluid antigen-specific antibody level and infection of $S$.

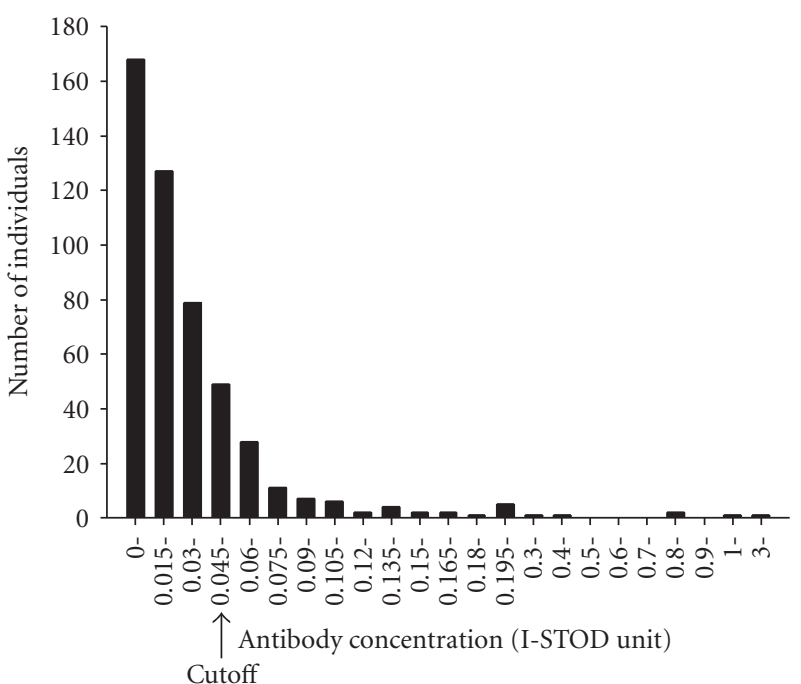

Figure 1: The distribution of Taenia solium cyst-fluid antigenspecific antibody concentration (I-STOD unit) in the study population. $Y$-axis: number of individuals; $X$-axis: antibody concentration by arbitrary I-STOD unit; arrow points to the positive cutoff value for seropositivity.

TABLE 2: Multivariate linear regression analysis between Taenia solium cyst-fluid antigen-specific antibody level and potential confounders.

\begin{tabular}{lccc}
\hline Variable & Coefficient & $P$ & $95 \%$ CI \\
\hline Age & -0.0007 & .350 & $-0.0021-0.0007$ \\
Sex & -0.0042 & .623 & $-0.0210-0.0126$ \\
S. japonicum infection & -0.0096 & .402 & $-0.0321-0.0129$ \\
Ascaris infection & 0.0058 & .529 & $-0.0122-0.0238$ \\
Trichuris infection & -0.0364 & .019 & $-0.0669-0.0060$ \\
Hookworm infection & -0.0065 & .431 & $-0.0227-0.0097$ \\
\hline
\end{tabular}

japonicum, A. lumbricoides, or Hookworm was detected. T. solium cyst-fluid antigen-specific antibody levels were lower in individuals with T. tricuria infection (Trichuriasis) compared to individuals without Trichuriasis $(P=.019)$ (Table 2).

3.2.2. Seroprevalence of Cysticercosis. The overall seroprevalence of cysticercosis in this study sample was $24.6 \%$ (95\% CI: $20.8 \% \sim 28.6 \%)$. Seroprevalence in different sex-age groups is shown in Figure 2. Seroprevalence was not related with sex, age, or infection with any of the four helminths species according to results of multivariate logistic regression analysis (Table 3).

\section{Discussion}

Cysticercosis is a long-standing problem in Latin America, a growing problem in Africa and Asia, and an emerging problem in developed countries [25]. A comprehensive estimate of the societal costs of T. solium cysticercosis for the Eastern Cape Province (ECP), South Africa, suggested that cysticercosis results in considerable monetary costs to 


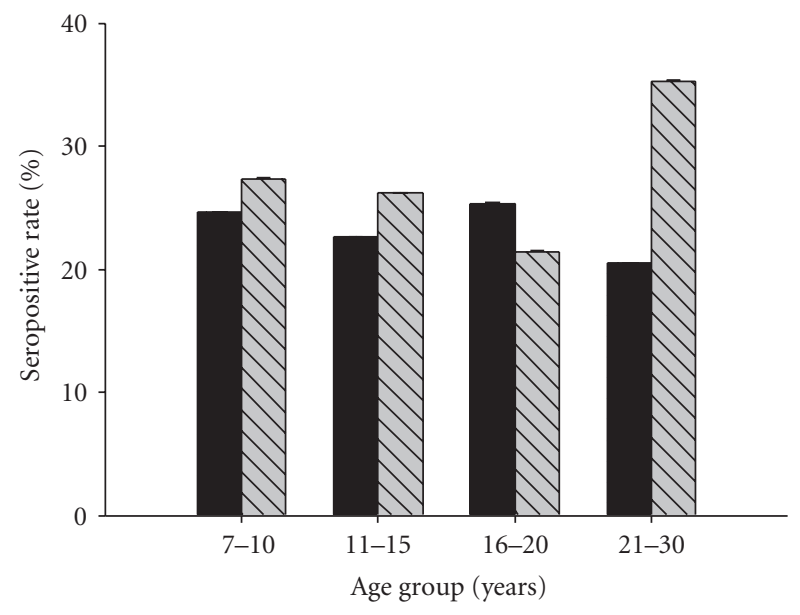

Male Female

Figure 2: Seroprevalence of cysticercosis in different sex-age groups. The numbers of subjects in different groups are as follows. Male: age 7-10 $(n=77)$, age $11-15(n=97)$, age 16-20 $(n=63)$, age 21-30 $(n=73)$; female: age $7-10(n=62)$, age $11-15(n=80)$, age $16-20(n=28)$, age $21-30(n=17)$. No statistical difference was found for seroprevalence of all groups $(P=.931)$.

TABLE 3: Multivariate logistic regression analysis between seroprevalence of cysticercosis and potential confounders.

\begin{tabular}{lccc}
\hline Variable & Coefficient & $P$ & $95 \%$ CI \\
\hline Age group & -0.0273 & .803 & $-0.2416-0.1870$ \\
Sex & 0.1249 & .578 & $-0.3149-0.5646$ \\
S. japonicum infection & -0.0103 & .972 & $-0.5941-0.5735$ \\
Ascaris infection & -0.1446 & .551 & $-0.6194-0.3302$ \\
Trichuris infection & -0.0504 & .900 & $-0.8361-0.7353$ \\
Hookworm infection & -0.2831 & .199 & $-0.7149-0.1486$ \\
\hline
\end{tabular}

a region that is already economically constrained [26]. A recent study on the disease burden of $T$. solium cysticercosis in Cameroon reported an average annual number of DALYs (Disability Adjusted Life Year) lost due to T. solium cysticercosis in West Cameroon amounting for 9.0 per thousand persons which is approximately 4 times higher than the same estimations already available for trypanosomiasis and schistosomiasis in sub-Saharan Africa [27].

In recent years, several epidemiological surveys of cysticercosis using both antigen and antibody detection methods have been carried out in endemic areas in Asia. These studies reported a seroprevalence of cysticercosis ranging from $2 \%$ to $48 \%$ in different districts in Indonesia, 5\%-7\% in Bac Ninh province of Vietnam, $0.02 \%-11.2 \%$ in different areas of China [16], and 3.2\%-42.6\% in Vellore district of south India [28].

In the Philippines, to date, there have been only 15 published cases of neurocysticercosis $[20,29,30]$. The prevalence of cysticercosis in pigs was $0.167 \%(8,276 / 4,956,422)$ based on direct examination of slaughtered pigs between 1970 and 1974 in twenty selected areas in the Philippines [16]. In 2005, veterinarians at the National Abattoir stated that in Leyte, the Philippines, there were still T. solium-infected animals; however, no data were provided to support this claim [31].

To our knowledge, ours is the first community-based study examining the seroprevalence of human cysticercosis in the Philippines. The seroprevalence of cysticercosis in our study sample was $24.6 \%$, although comparable with other surveys in Asia [16], the prevalence was considerably higher than expected, given the absence of documented reports of cysticercosis in this region. Even though antibody detection assays might overestimate current infection prevalence $[18,19]$, we believe that our data support cysticercosis as a significant public health problem consistent with the poor sanitation, and unpenned animal farming practices in the study site [21, 31]. Additional studies are necessary using other serology tests $[2,18]$ including antigen detection methods to confirm the high prevalence of cysticercosis in the study area, while neuroimaging (CT scan) of persons testing sero-positive with neurological problems would also be helpful to validate the serological findings.

We did not identify any subjects excreting Taenia eggs in stool by Kato-Katz exam. Given the high seroprevalence, this apparent paradox is in fact consistent with the epidemiology of transmission of T. solium [19, 32]. For example, 95\% of Indian patients with NCC were vegetarians or did not admit to consume pork [16]. The episodic nature of egg excretion $[1,4,32,33]$, coupled with the low sensitivity of microscopic stool examination likely contributed to this discrepancy [4]. Future work should include more sensitive egg detection methods such as a coproantigen-based diagnosis test [33].

Clinically, schistosomiasis japonica has been a main concern in the study area and has been implicated in the neurological complications of the residents [34-37]. In 1950, a study reported a prevalence of cerebral schistosomiasis of $2 \%$ among the American soldiers $(n=600)$ who were infected with S. japonicum from 1944 to 1945 in Leyte [38], supporting the estimate that cerebral involvement in schistosomiasis occurs in $2 \%$ to $5 \%$ of infected individuals [39]. The high prevalences of schistosomiasis and neuroschistosomiasis, coupled with a report suggesting that $T$. saginata infections are more common than $T$. solium infections in humans in the Philippines [16, 21], have contributed to the underappreciation of T. solium as a potential cause of neurological complications in the study area. The overlapping clinical features of cerebral schistosomiasis japonica [40] and NCC, together with the high seropositive proportion of cysticercosis in our study sample suggest that cysticercosis should remain in the differential diagnosis of seizure disorders in this region.

While our data supports an unexpectedly high estimate of cysticercosis prevalence in the study population, several study limitations merit discussion. First, our study population has a higher prevalence of $S$. japonicum infection compared to the general community. This sampling approach would lead to overestimating the community prevalence of cysticercosis if there are common environmental, genetic, or immunologic factors that predispose to both infections. We believe that our results are generalizable to non-S. 
japonicum infected populations because we did not detect a significantly different seroprevalence of cysticercosis in the S. japonicum infected $(24.2 \%, 102 / 422)$ compared to the S. japonicum uninfected $(26.7 \%, 20 / 75)$ individuals $(P=.643)$.

Second, the immunodiagnostic method employed in our study is based on indirect ELISA to detect specific IgG using T. solium cyst-fluid antigen and may be susceptible to crossreactivity to schistosome antibodies. This kit was used in the national epidemiological survey for cysticercosis during 2001-2003 in China [41]. Besides its documented sensitivity $(92.5 \%)$ and specificity (100\%), a later study reported a positive rate of $78 \%$ in 70 patients with cysticercosis using this kit [42]. These test characteristics support the validity of the seroprevalence data obtained in our study sample. Currently, there are no commercially available antibody detection kits for serodiagnosis of cysticercosis that have been specifically validated for false-positivity in S. japonicum infected individuals. We do not think that false-positive test results due to coinfections with other helminths contributed to our high seroprevalence because the seroprevalence for cysticercosis did not differ in individuals with and without these coinfections (see Tables 2 and 3 ).

Third, anticysticercal antibody may persist long after the parasite has been eliminated by either immune mechanisms or drug therapy [43]; and antibody may reflect exposure to parasite antigens rather than actual infection [44]. Thus, the detection method applied in the study could contribute to overestimation of the prevalence of active cysticercosis.

Fourth, the age range of our study sample was restricted to 7-30 years, which may have decreased our ability to detect a relationship between cysticercosis seropositivity and host age (Figure 2, Tables 2 and 3) as Fleury et al. observed [45].

Despite the limitations of antibody detection using cystfluid antigen, a high community seroprevalence for cysticercosis identifies a "hot spot" where preventive and control measures should be targeted. The high seroprevalence of human cysticercosis in the present study sample suggests that cysticercosis may be a significant, yet unrecognized problem in the Philippines. These pilot data in a selected sample underscore the need for community and school based surveys of cysticercosis as well as studies identifying risk factors for cysticercosis in rural areas of the Philippines. In addition, our results support a reexamination of pigs as a sentinel indicator for human cysticercosis in the same area [46].

\section{Acknowledgments}

This study was funded by Natural Science Foundation of China no. 30671836 and the NIH Grant no. AI48123. The authors thank the villagers in Macanip for their participation in this study and the field team workers for their help in sample collection and microscopic analysis. They also thank Dr Feng Chen at the Department of Statistics of Nanjing Medical University for assistance with data analysis, and Dr. Paul Knopf at Brown University for constructive discussions.

\section{References}

[1] A. Flisser, "Taeniasis and cysticercosis due to Taenia solium," Progress in Clinical Parasitology, vol. 4, pp. 77-116, 1994.

[2] R. Kraft, "Cysticercosis: an emerging parasitic disease," American Family Physician, vol. 76, no. 1, pp. 91-96, 2007.

[3] H. H. Garcia, R. Gilman, M. Martinez, et al., "Cysticercosis as a major cause of epilepsy in Peru," The Lancet, vol. 341, no. 8839, pp. 197-200, 1993.

[4] H. H. García, A. E. Gonzalez, C. A. W. Evans, and R. H. Gilman, "Taenia solium cysticercosis," The Lancet, vol. 362, no. 9383, pp. 547-556, 2003.

[5] T. L. Mac, D.-S. Tran, F. Quet, P. Odermatt, P.-M. Preux, and C. T. Tan, "Epidemiology, aetiology, and clinical management of epilepsy in Asia: a systematic review," Lancet Neurology, vol. 6, no. 6, pp. 533-543, 2007.

[6] S. M. Montano, M. V. Villaran, L. Ylquimiche, et al., "Neurocysticercosis: association between seizures, serology, and brain CT in rural Peru," Neurology, vol. 65, no. 2, supplement 1, pp. 229-234, 2005.

[7] G. Nsengiyumva, M. Druet-Cabanac, B. Ramanankandrasana, B. Bouteille, L. Nsizabira, and P.-M. Preux, "Cysticercosis as a major risk factor for epilepsy in Burundi, East Africa," Epilepsia, vol. 44, no. 7, pp. 950-955, 2003.

[8] A. L. Willingham III and D. Engels, "Control of Taenia solium cysticercosis/taeniosis," Advances in Parasitology, vol. 61, pp. 509-566, 2006.

[9] A. Carpio, "Neurocysticercosis: an update," Lancet Infectious Diseases, vol. 2, no. 12, pp. 751-762, 2002.

[10] Y. del la Garza, E. A. Graviss, N. G. Daver, et al., "Epidemiology of neurocysticercosis in Houston, Texas," American Journal of Tropical Medicine and Hygiene, vol. 73, no. 4, pp. 766-770, 2005.

[11] M. P. Earnest, L. B. Reller, C. M. Filley, and A. J. Grek, "Neurocysticercosis in the United States: 35 cases and a review," Reviews of Infectious Diseases, vol. 9, no. 5, pp. 961979, 1987.

[12] I. Mamkin, N. Sood, and S. V. Ramanan, "Taenia solium neurocysticercosis," New England Journal of Medicine, vol. 357, no. 16, pp. 1666-1667, 2007.

[13] F. J. Sorvillo, C. DeGiorgio, and S. H. Waterman, "Deaths from cysticercosis, United States," Emerging Infectious Diseases, vol. 13, no. 2, pp. 230-235, 2007.

[14] P. J. Hotez, M. E. Bottazzi, C. Franco-Paredes, S. K. Ault, and M. R. Periago, "The neglected tropical diseases of Latin America and the Caribbean: a review of disease burden and distribution and a roadmap for control and elimination," PLoS Neglected Tropical Diseases, vol. 2, no. 9, article e300, 2008.

[15] P. J. Hotez, "Neglected infections of poverty in the United States of America," PLoS Neglected Tropical Diseases, vol. 2, no. 6, article e256, 2008.

[16] V. Rajshekhar, D. D. Joshi, N. Q. Doanh, N. Van De, and Z. Xiaonong, "Taenia solium taeniosis/cysticercosis in Asia: epidemiology, impact and issues," Acta Tropica, vol. 87, no. 1, pp. 53-60, 2003.

[17] A. G. Diop, H. M. de Boer, C. Mandlhate, L. Prilipko, and H. Meinardi, "The global campaign against epilepsy in Africa," Acta Tropica, vol. 87, no. 1, pp. 149-159, 2003.

[18] P. Dorny, J. Brandt, A. Zoli, and S. Geerts, "Immunodiagnostic tools for human and porcine cysticercosis," Acta Tropica, vol. 87, no. 1, pp. 79-86, 2003.

[19] A. Flisser and T. W. Gyorkos, "Contribution of immunodiagnostic tests to epidemiological/intervention studies of 
cysticercosis/taeniosis in Mexico," Parasite Immunology, vol. 29, no. 12, pp. 637-649, 2007.

[20] M. A. G. Atilano and A. C. Pena, "Neurocysticercosis," Philippine Journal of Microbiology \& Infectious Diseases, vol. 29, pp. 41-47, 2000.

[21] P. V. Arambulo III, B. D. Cabrera, and M. S. Tongson, "Studies on the zoonotic cycle of Taenia saginata taeniasis and cysticercosis in the Philippines," International Journal of Zoonoses, vol. 3, no. 2, pp. 77-104, 1976.

[22] H. M. Coutinho, L. P. Acosta, H. W. Wu, et al., "Th2 cytokines are associated with persistent hepatic fibrosis in human Schistosoma japonicum infection," Journal of Infectious Diseases, vol. 195, no. 2, pp. 288-295, 2007.

[23] A. E. Ezeamama, S. T. McGarvey, L. P. Acosta, et al., "The synergistic effect of concomitant schistosomiasis, hookworm, and trichuris infections on children's anemia burden," PLoS Neglected Tropical Diseases, vol. 2, no. 6, article e245, 2008.

[24] J. Luo, J. Xu, Y. Zhang, et al., "I-STOD: a new standardization method for analysing indirect-ELISA results of a schistosomiasis field study," Parasitology, vol. 135, no. 4, pp. 453-465, 2008.

[25] Z. Pawlowski, J. Allan, and E. Sarti, "Control of Taenia solium taeniasis/cysticercosis: from research towards implementation," International Journal for Parasitology, vol. 35, no. 11-12, pp. 1221-1232, 2005.

[26] H. Carabin, R. C. Krecek, L. D. Cowan, et al., "Estimation of the cost of Taenia solium cysticercosis in Eastern Cape Province, South Africa," Tropical Medicine and International Health, vol. 11, no. 6, pp. 906-916, 2006.

[27] N. Praet, N. Speybroeck, R. Manzanedo, et al., "The disease burden of Taenia solium cysticercosis in Cameroon," PLoS Neglected Tropical Diseases, vol. 3, no. 3, article 406, 2009.

[28] V. Prabhakaran, M. V. Raghava, V. Rajshekhar, J. Muliyil, and A. Oommen, "Seroprevalence of Taenia solium antibodies in Vellore district, south India," Transactions of the Royal Society of Tropical Medicine and Hygiene, vol. 102, no. 3, pp. 246-250, 2008.

[29] M. Nakajima, K. Tashima, T. Hirano, F. Nakamura-Uchiyama, Y. Nawa, and M. Uchino, "A case of neurocysticercosis suggestive of a reinfection, 20 years after the initial onset," Rinsho Shinkeigaku, vol. 42, no. 1, pp. 18-23, 2002.

[30] D. Wada, M. Morita, and J. M. Hardman, "January 2004: elderly Filipino man with frontal lobe tumor," Brain Pathology, vol. 14, no. 3, pp. 337-338, 2004.

[31] W. U. de Leon, "Taeniasis and Cysticercosis in the Philippines," in Taeniasis/Cysticercosis and Echinococcosis in Asia, A. Ito, H. Wen, and H. Yamasaki, Eds., vol. 2 of Asian Parasitology Series Monograph, pp. 85-88, Federation of Asian Parasitologists, Chiba, Japan, 2005.

[32] E. Newell, F. Vyungimana, S. Geerts, I. Van Kerckhoven, V. C. W. Tsang, and D. Engels, "Prevalence of cysticercosis in epileptics and members of their families in Burundi," Transactions of the Royal Society of Tropical Medicine and Hygiene, vol. 91, no. 4, pp. 389-391, 1997.

[33] J. C. Allan and P. S. Craig, "Coproantigens in taeniasis and echinococcosis," Parasitology International, vol. 55, supplement, pp. S75-S80, 2006.

[34] F. J. Carod-Artal, "Neurological complications of Schistosoma infection," Transactions of the Royal Society of Tropical Medicine and Hygiene, vol. 102, no. 2, pp. 107-116, 2008.

[35] S. Jaureguiberry and E. Caumes, "Neurological involvement during Katayama syndrome," Lancet Infectious Diseases, vol. 8, no. 1, pp. 9-10, 2008.
[36] T. Leenstra, L. P. Acosta, G. C. Langdon, et al., "Schistosomiasis japonica, anemia, and iron status in children, adolescents, and young adults in Leyte, Philippines," American Journal of Clinical Nutrition, vol. 83, no. 2, pp. 371-379, 2006.

[37] J. E. H. Pittella, "Neuroschistosomiasis," Brain Pathology, vol. 7, no. 1, pp. 649-662, 1997.

[38] H. Most, C. A. Kane, P. H. Lavietes, et al., "Schistosomiasis japonica in American military personnel: clinical studies of 600 cases during the first year after infection," American Journal of Tropical Medicine and Hygiene, vol. 30, supplement 1, pp. 239-299, 1950.

[39] E. G. Garcia, "Clinical studies on schistosomiasis japonica in the Philippines: a review," Southeast Asian Journal of Tropical Medicine and Public Health, vol. 7, no. 2, pp. 247-256, 1976.

[40] M. Hayashi, H. Matsuda, L. C. Tormis, J. S. Nosenas, and B. L. Blas, "Clinical study on cerebral schistosomiasis japonica on Leyte island, Philippines: follow-up study 6 years after treatment with antischistosomal drugs," Southeast Asian Journal of Tropical Medicine and Public Health, vol. 15, no. 4, pp. 502-506, 1984.

[41] Y. Tu, X. Liu, and G. Li, "Analysis of clinical characters for 96 cysticercosis," Journal of Tropical Diseases and Parasitology, vol. 2, pp. 217-218, 2004.

[42] Z. Yuan, C. Zhou, J. Wang, Y. Chen, and L. Xu, "Comparison of difference among three groups of cysticercosis kit in the national survey on the important parasitic diseases," Chinese Journal of Parasitic Disease Control, vol. 18, p. 107, 2005.

[43] H. H. Garcia, R. H. Gilman, M. Catacora, et al., "Serologic evolution of neurocysticercosis patients after antiparasitic therapy," Journal of Infectious Diseases, vol. 175, no. 2, pp. 486489, 1997.

[44] H. H. Garcia, A. E. Gonzalez, R. H. Gilman, et al., "Short report: transient antibody response in Taenia solium infection in field conditions - a major contributor to high seroprevalence," American Journal of Tropical Medicine and Hygiene, vol. 65 , no. 1, pp. 31-32, 2001.

[45] A. Fleury, J. Morales, R. J. Bobes, et al., "An epidemiological study of familial neurocysticercosis in an endemic Mexican community," Transactions of the Royal Society of Tropical Medicine and Hygiene, vol. 100, no. 6, pp. 551-558, 2006.

[46] A. Flisser, R. Rodríguez-Canul, and A. L. Willingham III, "Control of the taeniosis/cysticercosis complex: future developments," Veterinary Parasitology, vol. 139, no. 4, pp. 283-292, 2006. 


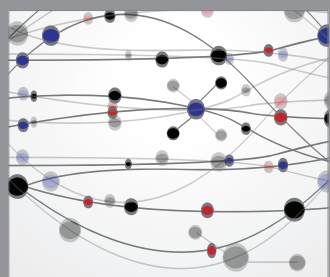

The Scientific World Journal
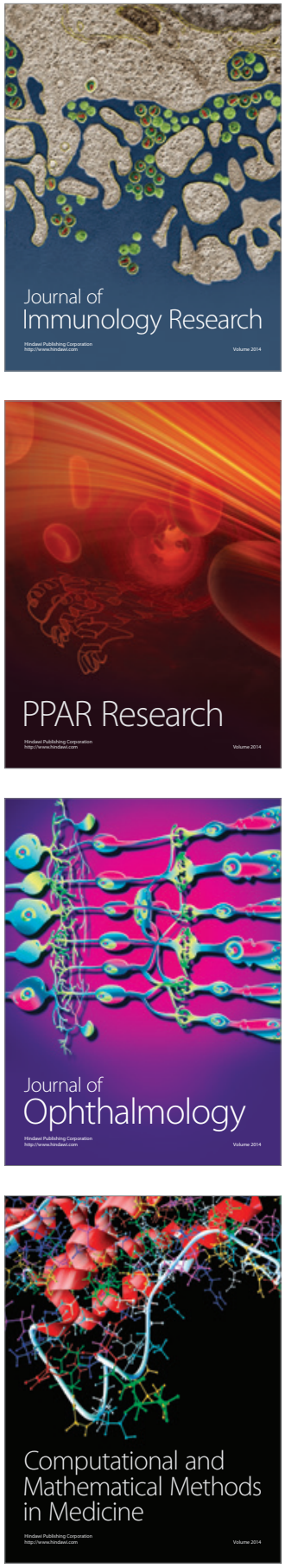

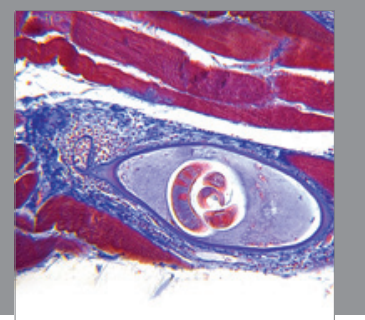

Gastroenterology

Research and Practice
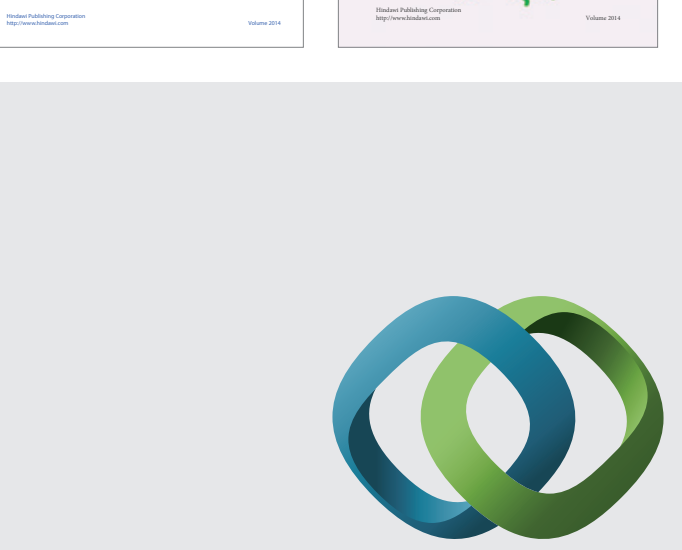

\section{Hindawi}

Submit your manuscripts at

http://www.hindawi.com
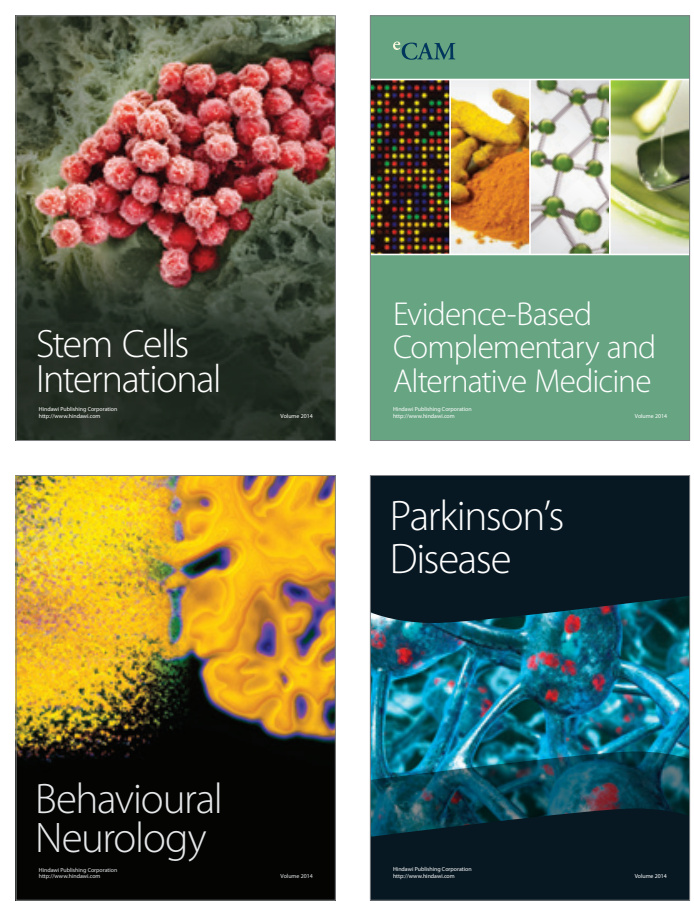

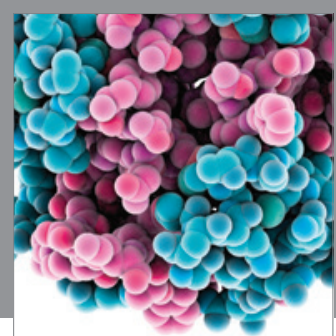

Journal of
Diabetes Research

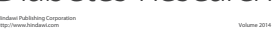

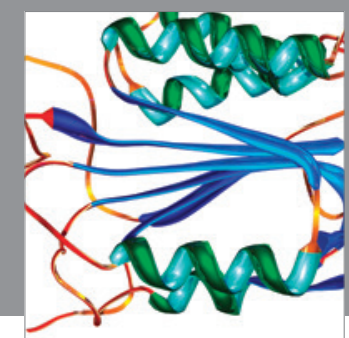

Disease Markers
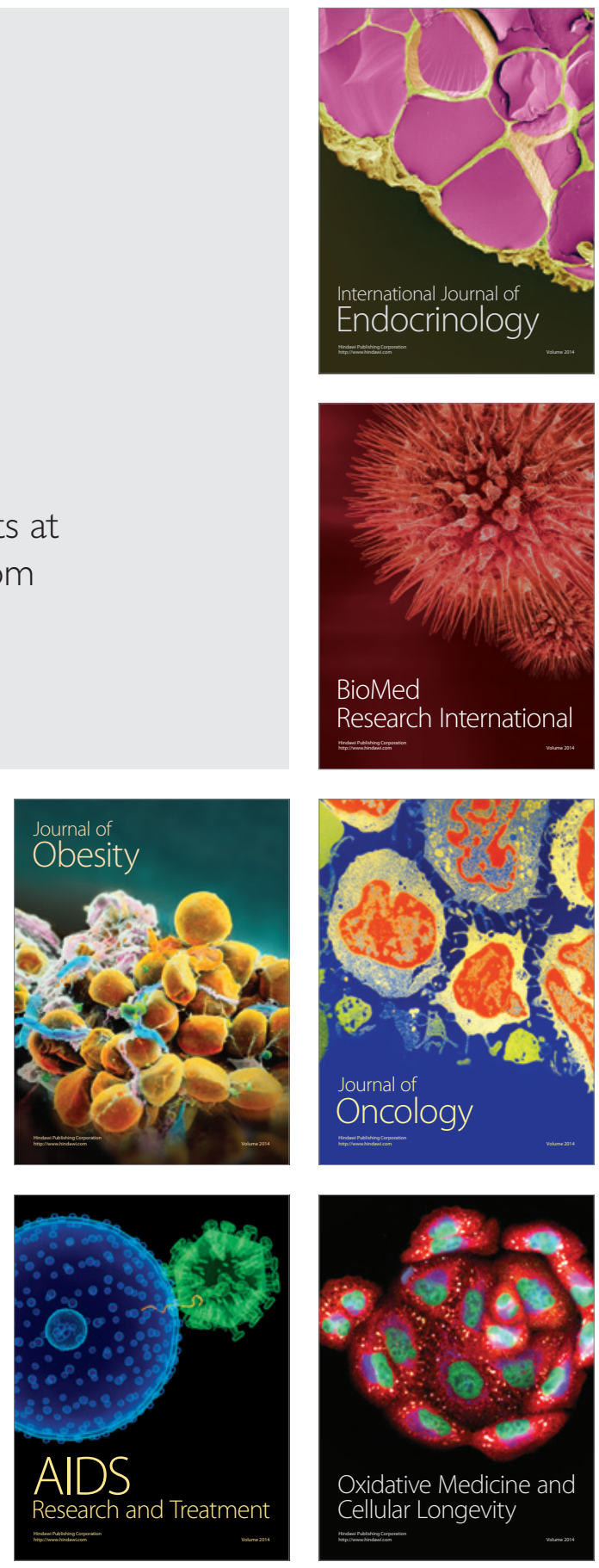